\title{
Galactic Structures from Gravitational Radii
}

\author{
Salvatore Capozziello ${ }^{1,2,3, *,+}$, Duško Borka ${ }^{4,+}$, Vesna Borka Jovanović ${ }^{4, \dagger}$ and Predrag Jovanović ${ }^{5, \dagger}$ \\ 1 Dipartimento di Fisica “E. Pancini”, Università di Napoli “Federico II", Complesso di Monte Sant'Angelo, \\ Edificio G, Via Cinthia, I-80126 Napoli, Italy \\ 2 Istituto Nazionale di Fisica Nucleare (INFN) Sezione di Napoli, Complesso di Monte Sant'Angelo, \\ Edificio G, Via Cinthia, I-80126 Napoli, Italy \\ 3 Gran Sasso Science Institute, Viale F. Crispi, 7, I-67100 L'Aquila, Italy \\ 4 Atomic Physics Laboratory (040), Vinča Institute of Nuclear Sciences, University of Belgrade, P.O. Box 522, \\ 11001 Belgrade, Serbia; dusborka@vinca.rs (D.B.); vborka@vinca.rs (V.B.J.) \\ 5 Astronomical Observatory, Volgina 7, P.O. Box 74, 11060 Belgrade, Serbia; pjovanovic@aob.rs \\ * Correspondence: capozzie@na.infn.it; Tel.: +39-081-676-496 \\ + These authors contributed equally to this work.
}

Received: 5 December 2017; Accepted: 2 January 2018; Published: 7 February 2018

\begin{abstract}
We demonstrate that the existence of a Noether symmetry in $f(R)$ theories of gravity gives rise to an additional gravitational radius, besides the standard Schwarzschild one, determining the dynamics at galactic scales. By this feature, it is possible to explain the baryonic Tully-Fisher relation and the rotation curve of gas-rich galaxies without the dark matter hypothesis. Furthermore, under the same standard, the Fundamental Plane of elliptical galaxies can be addressed.
\end{abstract}

Keywords: modified theories of gravity; methods: analytical; methods: numerical; galaxies; galaxies: fundamental parameters

\section{Introduction}

Our main motivation for this paper is to explain the observed galactic and extragalactic dynamics using gravitational potentials derived from Extended Gravity without dark matter (DM). There are two approaches for addressing extreme (weak field) gravity regime at galactic scales:

(i) using DM hypothesis in addition to Newtonian gravity (standard approach);

(ii) modifying fundamental laws of dynamics or gravity (alternative approach).

Regarding the latter approach, it was shown that MOND is able to successfully explain the dynamics of galaxies outside of galaxy clusters and a recently discovered tight relation between the radial acceleration inferred from their observed rotation curves and the acceleration due to the baryonic components of their disks (McGaugh et al. [1]). Some other theories of modified gravity which give the weak field point particle gravitational potential with Yukawa correction term are also able to explain flat rotation curves (see e.g., Brownstein \& Moffat [2]). Recently, Israel \& Moffat [3] showed that generalized Scalar-Tensor-Vector-Gravity (STVG) theory can also explain dynamics of the merging galaxy clusters, such as Abell 520 and the Bullet Cluster, without dark matter. This approach, however, requires modification of General Relativity (GR) and derivation of alternative field equations, as well as their cosmological reformulation for Friedmann-Lemaitre-Robertson-Walker metric and perfect fluid-the Friedmann equations.

Here we will try to explain the possible new fundamental gravitational radii which play analogue role in the case of weak gravitational field at galactic scales, as the Schwarzschild radius for strong gravitational field in the vicinity of some massive object (we have IR and UV gravitational radii).

New gravitational radii come from the extra degrees of freedom of Extended Gravity. 
In Section 2 we explain the fundamental plane (FP) of elliptical galaxies, and in Section 3 we explain extragalactic phenomena, such as the baryonic Tully-Fisher relation (BTFR) of gas-rich galaxies, all without the DM hypothesis.

\section{Fundamental Plane of Elliptical Galaxies and $f(R)$ Gravity}

\subsection{Fundamental Plane of Ellipticals}

It is well known that besides the spiral galaxies, elliptical galaxies could also have so called missing mass problem, where an extra mass is required to explain the observed differences between their dynamical masses and luminosities. The two possibilities explaining this missing mass problem would be dark matter (DM), or theories of modified gravity. As here we adopt the second approach, we study whether the $f(R)$ gravity could solve the missing mass problem in elliptical galaxies without dark matter hypothesis. We adopt such an approach because in the galaxies we can deal with the extreme gravity regimes, and higher order curvature corrections in gravity action can emerge. That is why instead of GR we will use its simplest extension: $f(R)$ gravity model.

Many characteristics of normal elliptical galaxies are correlated, which has been empirically shown. For example, a galaxy with a higher luminosity has a larger effective radius. Besides, more luminous elliptical galaxies have larger central velocity dispersions. A set of correlations connecting the global properties of elliptical galaxies is called fundamental plane (FP), and it is an empirical relation [4]:

$$
\log \left(r_{e}\right)=a \times \log \left(v_{c}\right)+b \times \log \left(I_{e}\right)+c,
$$

with: $r_{e}$-effective or half-light radius (encloses half of the total luminosity emitted by a galaxy), $v_{c}$-central velocity dispersion, $I_{e}$-mean surface brightness within the effective radius. We illustrate this region of parameter space in Figures 1-4. In this sense, some object can be represented as a point in the parameter space $r_{e}, v_{\mathcal{c}}, I_{e}$.

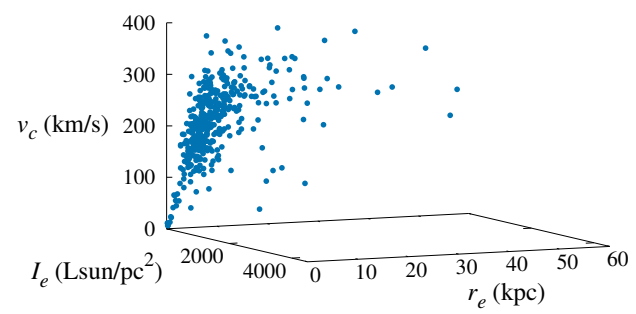

Figure 1. The three parameters of the fundamental plane (FP): surface brightness $I_{e}$, effective radius $r_{e}$ and circular velocity $v_{\mathcal{C}}$, for a sample of elliptical galaxies listed in Table 1 from [5]. Note: in paper [5] it is printed the first page only, and we used the whole sample of 401 ellipticals, available among the source files of its arxiv version.



Figure 2. The same as Figure 1, but from different view. 


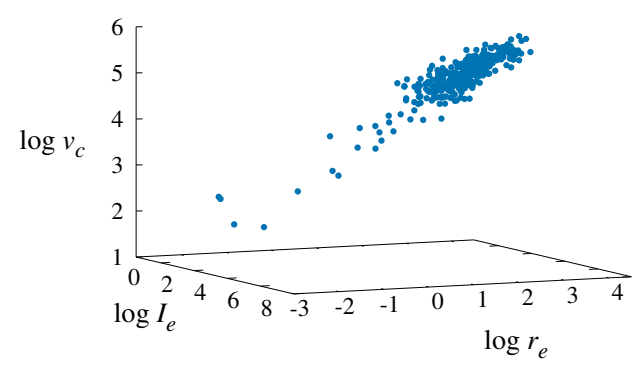

Figure 3. The FP parameter space from Figure 1, presented by logarithms of parameter values: $\log I_{e}$, $\log r_{e}$ and $\log v_{c}$.

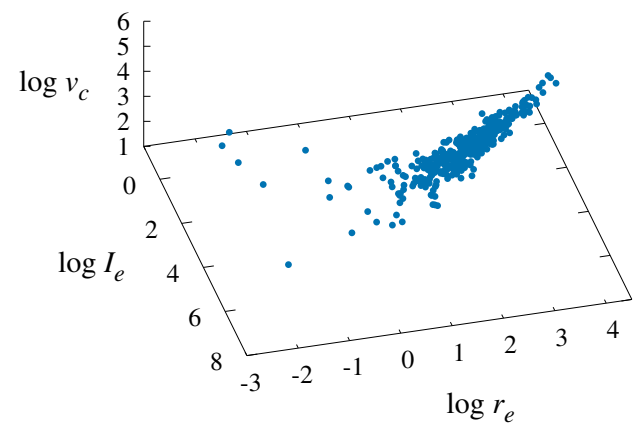

Figure 4. The same as Figure 3, but from different view.

\section{2. $f(R)$ Gravity and Dynamics of Stellar Systems}

We adopt $f(R)$ gravity which is the straightforward generalization of Einstein's General Relativity as soon as the function is $f(R) \neq \mathrm{R}$, that is, it is not linear in the Ricci scalar $R$ as in the Hilbert-Einstein action. $R^{n}$ gravity is the power-law version of $f(R)$ modified gravity. In the weak field limit, its potential (generated by a pointlike mass $m$ at the distance $r$ ) is [6]:

$$
\Phi(r)=-\frac{G m}{2 r}\left[1+\left(\frac{r}{r_{c}}\right)^{\beta}\right]
$$

where $r_{c}$ is scalelength depending on the gravitating system properties and $\beta$ is universal constant which depends on power $n$ :

$$
\beta=\frac{12 n^{2}-7 n-1-\sqrt{36 n^{4}+12 n^{3}-83 n^{2}+50 n+1}}{6 n^{2}-4 n+2} .
$$

Regarding the consideration of the power-law fourth-order theories of gravity, and determining the space parameters of $f(R)$ gravity, see [6-11].

We want to show the connection of the fundamental plane of elliptical galaxies with $R^{n}$ gravity potential, by showing the correlation between the corresponding parameters. In paper [12] the empirical result for coefficients $a$ and $b$ is obtained: $a=1.4, b=-0.85$. The test for our method is to recover these coefficients, starting from the gravitational potential derived from $f(R)$ gravity. Recovering FP using $f(R)$ gravity, which means finding connection between the parameters of FP equation and parameters of the potential in Equation (2), we have done in the following way:

1. addend with $r_{e}$ : correlation between $r_{e}$ and $r_{c}\left(r_{c}\right.$-from $R^{n}$ potential)

2. addend with $\sigma_{0}$ : correlation between $\sigma_{0}$ and $v_{v i r}\left(v_{v i r}\right.$-virial velocity in $\left.R^{n}\right)$

3. addend with $I_{e}$ : correlation between $I_{e}$ and $r_{e}$ (through the $r_{c} / r_{e}$ ratio).

In this sense, we showed that the three addends of FP have to be connected to $f(R)$ parameters (see [11] for more details). 
We use the data given in Table 1 of [5], which represents the result of the collected efforts of many astronomers over the years (available in ASCI format among the source files of its arxiv version: https: / / arxiv.org/format/astro-ph/9707037, see 'metaplanetab1'). From that table we used values from the following columns: column (5) $\log v_{c}$ (we get $v_{c}$ in $\left.\mathrm{km} / \mathrm{s}\right)$, column (6) $\log \sigma_{0}(\mathrm{~km} / \mathrm{s})$, column (7) $\log r_{e}(\mathrm{kpc})$ and column (8) $\log I_{e}\left(\mathrm{~L}_{s u n} / \mathrm{pc}^{2}\right)$. For elliptical galaxies, the circular velocity inside effective radius is $v_{\mathcal{c}}\left(r_{e}\right)=\sigma_{0}$, while for other stellar systems it is $v_{\mathcal{c}} \neq \sigma_{0}$.

The theoretical circular velocity we calculated for extended spherically symmetric systems, using Equation (25) from [6], given as a sum of Newtonian contribution $\left(v_{c, N}^{2}(r)\right.$-Newtonian rotation curve) and the correction term from $f(R)$ :

$$
v_{c}^{2}(r)=\frac{v_{c, N}^{2}(r)}{2}+\frac{r}{2} \frac{\partial \Phi_{c}}{\partial r},
$$

and taking into account the so called Hernquist profile for density distribution [13]:

$$
\rho(r)=\frac{a_{H} M}{2 \pi r\left(r+a_{H}\right)^{3}}, \quad a_{H}=\frac{r_{e}}{1+\sqrt{2}} .
$$

We fitted FP coefficients $a, b, c$ to the observed values $r_{e}, I_{e}$ and our calculated value $v_{c}^{\text {theor }}$, for different values of $r_{c} / r_{e}$ and $\beta$. One example of this fit we give in Figure 5 (with linear scale for $r_{e}$ ) and Figure 6 (with $\log$ scale for $r_{e}$ ). The coefficient $a$ is exactly like in [12], while $b$ has similar but not exactly the same value. However, we only calculated $v_{c}^{\text {theor }}$, while for $I_{e}$ we considered observed values, but in any case the agreement with data is very good.

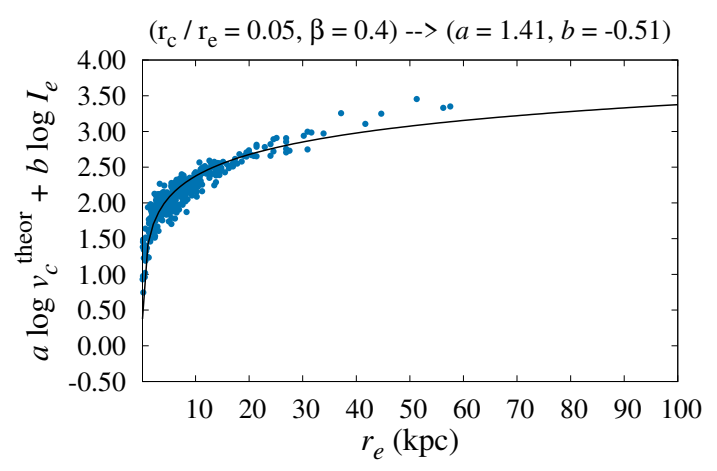

Figure 5. Fundamental plane (linear scale for $x$-axes) of elliptical galaxies with calculated circular velocity $v_{c}^{\text {theor }}$, and observed effective radius $r_{e}$ and mean surface brightness (within the effective radius) $I_{e}$, for $r_{c} / r_{e}=0.05$ and $\beta=0.4$. Black solid line is result of 3D fit of FP (calculated FP coefficients are $a=1.41$ and $b=-0.51$ ).

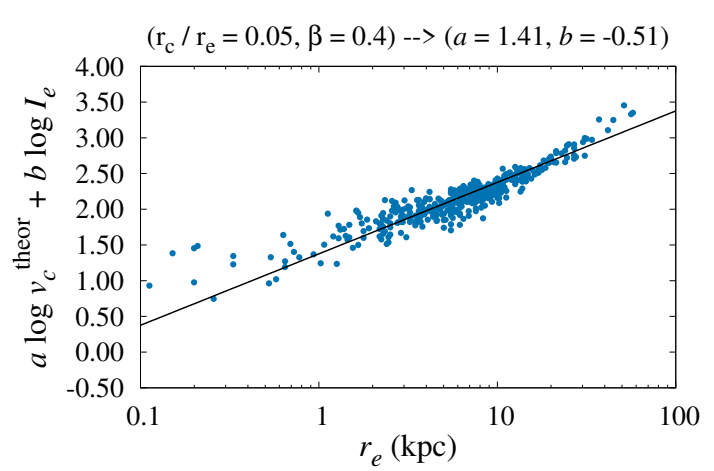

Figure 6. The same as Figure 5, but with log scale for $x$-axes. 
We obtained that the characteristic radius $r_{c}$ of $R^{n}$ gravity is proportional to the effective radius $r_{e}$ (more precise, $r_{c} \approx 0.05 r_{e}$ gives the best fit with data). This fact points out that the gravitational corrections induced by $R^{n}$ can lead photometry and dynamics of the system.

\section{Explaining the Baryonic Tully-Fisher Relation with New Gravitational Radius}

Some indications that $R^{n}$ gravity can explain the rotation curves of spiral galaxies, i.e., that the rotation curve of spiral galaxies could be fitted using the luminous components only, thus eliminating the need for dark matter, are argued in [6]. The authors investigated the possibility that the observed flatness of the rotation curves of spiral galaxies is not evidence for the existence of dark matter haloes, but rather a signal of the breakdown of General Relativity. In [14] it is found a reasonable agreement between the observed rotation curves of spiral galaxies and the circular velocity model in the framework of $R^{n}$ gravity, without the need for dark matter also. Due to the relevance of their sample, which contains objects in a large range of luminosities and with very accurate and proper kinematics, these results encouraged further investigations from both observational and theoretical points of view.

\subsection{New Fundamental Gravitational Radius}

Circular velocity of a point mass, in the $R^{n}$ gravity potential, can be found in the standard way, that is $v_{c}^{2}(r)=r \frac{d \Phi}{d r}$, which gives (for a detailed explanation see [6]):

$$
v_{c}^{2}(r)=\frac{G M}{2 r}\left[1+(1-\beta)\left(\frac{r}{r_{c}}\right)^{\beta}\right] .
$$

Characteristic length $r_{c}$ of $R^{n}$ gravity can be related to the MOND acceleration constant $a_{0}$ (for details see [15]):

$$
r_{c}=\sqrt{\frac{G M}{a_{0}}}
$$

Therefore, $r_{c}$ represents a new fundamental gravitational radius in the case of weak gravitational field.

\subsection{Tully-Fisher Relation in MOND and $f(R)$ Gravity}

Baryonic Tully-Fisher relation is empirical relation between galaxy mass and rotation velocity:

$$
M_{b} \sim v_{c}^{4}
$$

where $M_{b}$-baryonic mass: $M_{b}=M_{*}+M_{g}$ (star masses + gas mass), and $v_{c}$-circular velocity (after some radius $r_{f}$, we can assume that it becomes constant $v_{c} \approx v_{f}$ ).

The observational data for Figure 2 from [16], which shows BTFR, are given at the internet address http://astroweb.case.edu/ssm/data/gasrichdatatable.txt. These are collected data from references: [17-19]. In order to compare the BTF relations in MOND, $R^{n}$ and $\Lambda C D M$, we used these observational data, and we draw these lines at the same $M_{b}\left(v_{f}\right)$ graph:

(i) $\quad$ MOND: $M_{b}=\frac{v_{f}^{4}}{G a}$;

(ii) $R^{n}: M_{b}=\frac{4 a v_{f}^{4}}{G a_{f}^{2}\left(1+(1-\beta)\left(\frac{a}{a_{f}}\right)^{\beta}\right)^{2}}$;

We calculated BTFR for four cases of $n$ : $\frac{5}{4}, \frac{3}{2}, 2, \frac{7}{2}$, which correspond to $\beta=$ $0.358,0.518,0.667,0.817$, respectively. $a_{0}-\mathrm{MOND}$ acceleration constant for point source in 
infinity; $a$-constant for spiral systems. In the case of spiral galaxies, we have $a$ instead of $a_{0}$ (empirical calibration is $a_{0}=0.8 a$, as reported in [20]).

(iii) $\Lambda$ CDM: $M_{b}=0.17 M_{\text {vir }}, v_{f}=v_{\text {vir }}$.

Formula for $\Lambda \mathrm{CDM}$ is taken from the paper [21], Equation (13): $M_{\text {vir }}=\left(4.6 \times 10^{5} M_{\text {sun }} \mathrm{km}^{-3} \mathrm{~s}^{3}\right) v_{\text {vir }}^{3}$.

We show comparison between the observed and best fit baryonic Tully-Fisher relations of gas-rich galaxies in MOND, $R^{n}$ gravity (for four values of $\beta$ ) and $\Lambda C D M$, in Figures 7 and 8.

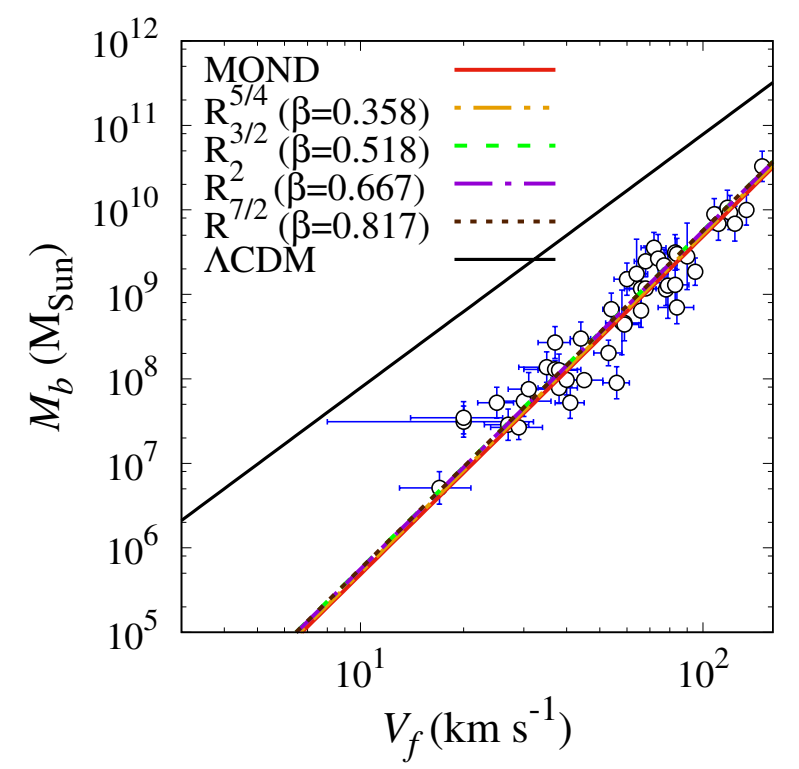

Figure 7. Comparison between best fit baryonic Tully-Fisher (BTF) relations of gas-rich galaxies (for a sample of galaxies used in [16]), in MOND, $R^{n}$ gravity for values of $n=1.25,1.5,2$ and 3.5 (corresponding $\beta$ are $0.358,0.518,0.667$ and 0.817 , respectively) and $\Lambda$ CDM. Note: all values were calculated by us, except for open circles which are observed data from [16].
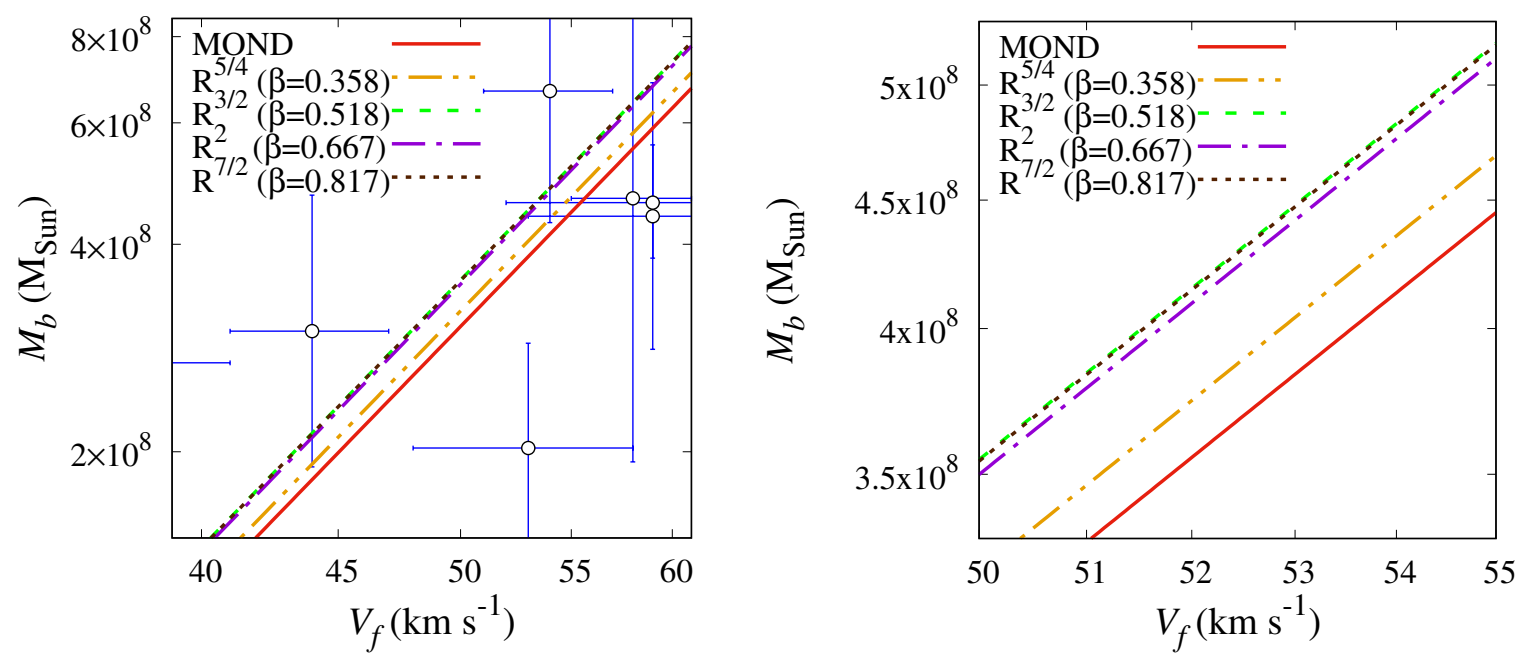

Figure 8. Left: one zoomed part of the Figure 7, showing BTF relations, in MOND and $R^{n}$ gravity.

Right: a zoomed part of the figure, for even smaller range of $V_{f}$ and $M_{b}$.

Using the fact that the weak field limit of $f(R)$ power-law gravity gives MOND as a particular case, and as it can be seen from the equations in this subsection, the following form of BTF relation in $R^{n}$ gravity can be recovered: $M_{b}=A(\beta) v_{f}^{4}$. The only difference in respect to $\operatorname{MOND}\left(M_{b}=A v_{f}^{4}\right)$ is that normalization factor $A$ depends on universal constant $\beta$ of $R^{n}$ gravity. 


\section{Discussion and Conclusions}

We showed that it is possible to explain the baryonic Tully-Fisher relation, the rotation curve of gas-rich galaxies, and the features of fundamental plane of ellipticals without the dark matter hypothesis.

We can summarize our conclusions like this:

- We used power-law $f(R)$ gravity to demonstrate the existence of a new fundamental gravitational radius.

- This radius plays an analog role, in the case of weak gravitational field at galactic scales (IR scales) as the Schwarzschild radius in the case of strong gravitational field in the vicinity of compact massive objects (UV scales).

- The radius emerges as a conserved quantity from Noether's symmetries that exist for any power-law $f(R)$ function.

- Using this new gravitational radius, $f(R)$ gravity is able to explain the baryonic Tully-Fisher relation of gas-rich galaxies without DM hypothesis.

- $\quad$ MOND is a particular case of $f(R)$ gravity in the weak field limit.

- The same radius is useful to address the FP of elliptical galaxies.

- The range $0.5 \leq \beta \leq 0.8$ (corresponding to $1.5 \leq n \leq 3.5$ ) is in a good agreement with observations. These values agree with observational constraints on $\beta$ obtained by fitting FP and MOND. We do not need DM to explain baryonic Tully-Fisher relation, and even more, $\Lambda$ CDM is not in satisfactory agreement with observations.

- For elliptical galaxies $r_{c}$ is proportional to $r_{e}$.

- Considering the definition of $r_{e}$, we can say that the effective radius (defined photometrically as the radius containing half of the luminosity of a galaxy) is led by gravity.

- In perspective, the whole galactic dynamics can be addressed by Extended Gravity.

- Work in progress for Faber-Jackson relation, galactic potentials, Boltzmann-Vlasov relation, and Virial Theorem.

Acknowledgments: This work is supported by Istituto Nazionale di Fisica Nucleare, Sezione di Napoli, Italy, iniziative specifiche TEONGRAV and QGSKY, and by Ministry of Education, Science and Technological Development of the Republic of Serbia, through the project 176003 "Gravitation and the Large Scale Structure of the Universe". The authors also acknowledge the support by Bilateral cooperation between Serbia and Italy 451-03-01231/2015-09/1 "Testing Extended Theories of Gravity at different astrophysical scales", of the COST Action CA15117 (CANTATA), supported by COST (European Cooperation in Science and Technology), and ERASMUS+ Programme (for higher education student and staff mobility) between Dipartimento di Fisica, Università degli studi di Napoli “Federico II" and Vinča Institute of Nuclear Sciences, University of Belgrade.

Author Contributions: All the coauthors participated in calculation and discussion of obtained results.

Conflicts of Interest: The authors declare no conflict of interest.

\section{Abbreviations}

The following abbreviations are used in this manuscript:

$\begin{array}{ll}\text { BTFR } & \text { Baryonic Tully-Fisher relation } \\ \text { DM } & \text { Dark matter } \\ \text { ETGs } & \text { Extended Theories of Gravity } \\ \text { FP } & \text { Fundamental plane } \\ \text { GC } & \text { Galactic Center } \\ \text { GR } & \text { General Relativity } \\ \text { MOND } & \text { Modified Newtonian dynamics } \\ \text { STVG } & \text { Scalar-Tensor-Vector-Gravity }\end{array}$




\section{References}

1. McGaugh, S.S.; Lelli, F.; Schombert, J.M. Radial acceleration relation in rotationally supported galaxies. Phys. Rev. Lett. 2016, 117, 201101.

2. Brownstein, J.R.; Moffat, J.W. The Bullet Cluster 1E0657-558 evidence shows modified gravity in the absence of dark matter. Mon. Not. R. Astron. Soc. 2007, 382, 29-47.

3. Israel, N.S.; Moffat, J.W. The Train Wreck Cluster Abell 520 and the Bullet Cluster 1E0657-558 in a Generalized Theory of Gravitation. arXiv 2016, arXiv:1606.09128.

4. Busarello, G.; Capaccioli, M.; Capozziello, S.; Longo, G.; Puddu, E. The relation between the virial theorem and the fundamental plane of elliptical galaxies. Astron. Astrophys. 1997, 320, 415-420.

5. Burstein, B.; Bender, R.; Faber, S.M.; Nolthenius, R. Global relationships among the physical properties of stellar systems. Astron. J. 1997, 114, 1365-1392.

6. Capozziello, S.; Cardone, V.F.; Troisi, A. Low surface brightness galaxy rotation curves in the low energy limit of $R^{n}$ gravity: No need for dark matter? Mon. Not. R. Astron. Soc. 2007, 375, 1423-1440.

7. Borka, D.; Jovanović, P.; Borka Jovanović, V.; Zakharov, A.F. Constraints on $R^{n}$ gravity from precession of orbits of S2-like stars. Phys. Rev. D 2012, 85, 124004.

8. Borka, D.; Jovanović, P.; Borka Jovanović, V.; Zakharov, A.F. Orbital precession in $R^{n}$ gravity: Simulations vs observations (the S2 star orbit case). In Proceedings of the Summer School and Conference on Modern Mathematical Physics, Belgrade, Serbia, 9-19 September 2012; Sveske fizičkih nauka (SFIN) 2013, XXVI Series A: Conferences No. A1, pp. 61-66.

9. Zakharov, A.F.; Borka, D.; Borka Jovanović, V.; Jovanović, P. Constraints on $R^{n}$ gravity from precession of orbits of S2-like stars: A case of a bulk distribution of mass. Adv. Space Res. 2014, 54, 1108-1112.

10. Borka, D.; Jovanović, P.; Borka Jovanović, V.; Zakharov, A.F. Chapter 9 in "Advances in General Relativity Research"; Williams, C., Ed.; Nova Science Publishers: Hauppauge, NY, USA, 2015; pp. 343-362.

11. Borka Jovanović, V.; Capozziello, S.; Jovanović, P.; Borka, D. Recovering the fundamental plane of galaxies by $f(R)$ gravity. Phys. Dark Univ. 2016, 14, 73-83.

12. Bender, R.; Burstein, D.; Faber, S.M. Dynamically hot galaxies. I. Structural properties. Astrophys. J. 1992, 399, 462-477.

13. Hernquist, L. An analytical model for spherical galaxies and bulges. Astrophys. J. 1990, 356, 359-364.

14. Frigerio Martins, C.; Salucci, P. Analysis of rotation curves in the framework of $R^{n}$ gravity. Mon. Not. R. Astron. Soc. 2007, 381, 1103-1108.

15. Capozziello, S.; Jovanović, P.; Borka Jovanović, V.; Borka, D. Addressing the missing matter problem in galaxies through a new fundamental gravitational radius. J. Cosmol. Astropart. Phys. 2017, 6, 044.

16. McGaugh, S.S. A Novel Test of the Modified Newtonian Dynamics with Gas Rich Galaxies. Phys. Rev. Lett. 2011, 106, 121303.

17. Begum, A.; Chengalur, J.N.; Karachentsev, I.D.; Sharina, M.E. Baryonic Tully-Fisher relation for extremely low mass Galaxies. Mon. Not. R. Astron. Soc. 2008, 386, 138-144.

18. Stark, D.V.; McGaugh, S.S.; Swaters, R.A. A first attempt to calibrate the baryonic Tully-Fisher relation with gas-dominated galaxies. Astron. J. 2009, 138, 392-401.

19. Trachternach, C.; de Blok, W.J.G.; McGaugh, S.S.; van der Hulst, J.M.; Dettmar, R.-J. The baryonic Tully-Fisher relation and its implication for dark matter halos. Astron. Astrophys. 2009, 505, 577-587.

20. McGaugh, S.S. Erratum: Novel Test of Modified Newtonian Dynamics with Gas Rich Galaxies. Phys. Rev. Lett. 2011, 107, 229901.

21. McGaugh, S.S. The baryonic Tully-Fisher relation of gas-rich galaxies as a test of $\Lambda$ CDM and MOND. Astron. J. 2012, 143, 40 .

(C) 2018 by the authors. Licensee MDPI, Basel, Switzerland. This article is an open access article distributed under the terms and conditions of the Creative Commons Attribution (CC BY) license (http://creativecommons.org/licenses/by/4.0/). 